\title{
Quantum interference with molecules: The role of internal states
}

\author{
Mark Hillery ${ }^{1,2}$, Leonard Mlodinow ${ }^{1 *}$, and Vladimír Bužek ${ }^{2,3}$ \\ 1 Department of Physics, Hunter College of CUNY, 695 Park Avenue, New York, NY 10021 USA \\ ${ }^{2}$ Research Center for Quantum Information, Slovak Academy of Sciences, 84511 Bratislava, Slovakia \\ ${ }^{3}$ Quniverse, Liščie údolie 116, 84104 Bratislava, Slovakia
}

\begin{abstract}
Recent experiments have shown that fullerene and fluorofullerene molecules can produce interference patterns. These molecules have both rotational and vibrational degrees of freedom. This leads one to ask whether these internal motions can play a role in degrading the interference pattern. We study this by means of a simple model. Our molecule consists of two masses a fixed distance apart. It scatters from a potential with two or several peaks, thereby mimicking two or several slit interference. We find that in some parameter regimes the entanglement between the internal states and the translational degrees of freedom produced by the potential can decrease the visibility of the interference pattern. In particular, different internal states correspond to different outgoing wave vectors, so that if several internal states are excited, the total interference pattern will be the sum of a number of patterns, each with a different periodicity. The overall pattern is consequently smeared out. In the case of two different peaks, the scattering from the different peaks will excite different internal states so that the path the molecule takes become entangled with its internal state. This will also lead to degradation of the interference pattern. How these mechanisms might lead to the emergence of classical behavior is discussed.
\end{abstract}

\section{INTRODUCTION}

How big does an object have to be before quantum mechanical effects disappear? A cat is presumably too large, but a molecule may not be. Interference with $\mathrm{H}_{2}$ molecules was demonstrated as early as 1930 [1], and there have been a number of subsequent experiments with small molecules [2-6]. In a series of recent experiments, the group at the University of Vienna has shown that molecules consisting of many atoms, such as fullerenes or fluorofullerenes can produce an interference pattern after travelling through a grating $[7,8]$. Molecules, however, come in many sizes, so one might expect that, in regard to their translational degrees of freedom, small molecules behave quantum mechanically while large ones do not. Where is the boundary between these behaviors, and what causes the transition, if indeed it takes place?

There are several mechanisms that can destroy an interference pattern produced by matter waves. For example, a decoherence of molecule in a beam can be caused by to collisions with lighter particles from the environment (see e.g. Refs. $[9,10]$ ). Other destructive environmental influences are grating vibrations, the finite size of a grating, or even Coriolis forces (see e.g. Ref. [11]). Obviously, the chief destroyer of quantum coherences is thermal radiation through which the molecules become entangled with external (light) degrees of freedom. This mechanism, which leads to disappearance of the interference pattern, has been studied theoretically by several groups. The interference pattern found by the group in Vienna disappears if the internal temperature of the molecules is sufficiently high (2000 K) [12]. This experimental result has been analyzed in detail and theoretically explained by K. Hornberger et al. [10,13]. In particular, these authors have studied the effect of thermal radiation at different temperatures of the molecule on the decoherence of fullerenes, taking into account that these molecules are not black body radiators. Under this assumption they have been able to find a good agreement with experimental results ${ }^{1}$.

Another possible source of decoherence is the coupling between the translational motion of the molecule and its internal states [10]. The vibrational and rotational states of the molecule can be thought of as a reservoir that the molecule carries with it. When the molecule passes through a region in which there is a potential, the translational motion of the molecule and its internal modes can become entangled. Therefore, the molecule's internal reservoir can

\footnotetext{
*Permanent address: Alexei Nicolai Inc., 1970 La France Avenue, South Pasadena, CA 91030

${ }^{1}$ In other studies (see e.g. Refs. [14,15]) an assumption has been made, that the molecule is a black-body radiator and consequently these results, while they illustrate the effect of this type of decoherence, do not apply directly to experiments in Vienna.
} 
cause different outgoing momenta or different paths the molecule can take through the region of nonzero potential to decohere.

Here we wish to examine the effect of the internal states of the molecule on its interference. We shall do so by considering a simple model that is a version of two-slit interference, the most fundamental quantum mechanical interference phenomenon. Our molecule will be a rigid rotator, consisting of two equal masses separated by a fixed distance, and, for simplicity, it will be confined to two dimensions. The molecule has both translational and rotational degrees of freedom. It will scatter off of a potential, which will initially be taken to be one consisting of two peaks (see Fig. 1). The molecule will interact with both parts of the potential, and the two scattered waves will both be incident on a detector at some distant point. The result of this process is described by the scattering cross section, and we shall be interested in whether or not it exhibits interference fringes. It is straightforward to extend our model to the case in which the potential has more than two peaks, in particular to the experimentally relevant situation in which it represents a diffraction grating, and interference pattern resulting from the scattering from this type of potential will be studied as well.

Entanglement between internal and translational states can, in fact, contribute to washing out an interference pattern, but only in certain parameter regimes. We shall examine two ways in which this can happen. The first is a result of the fact that after the scattering, different internal states of the molecule have different wave vectors describing their translational motion, i.e. the outgoing momenta and the internal states of the molecule become entangled. For example, if the molecule is initially in a plane wave state of its translational motion and not rotating, and the outgoing state contains components that correspond to rotational motion of the molecule, then these components will have a wave vector whose magnitude is smaller than that of the original wave vector. This follows from energy conservation; some of the translational kinetic energy has been transformed into rotational kinetic energy by the scattering. This mechanism was proposed by Hegerfeldt and Köhler as a means of separating an excited state of a molecule from its ground state [16]. Molecules, which either have or have not been excited, pass through a transmission grating, and, because of the difference in their wave vectors, the ground and excited state molecules scatter in different directions. In our case, the different parts of the state of the scattered molecule, each part having a different wave vector, result in a total interference pattern made up of patterns with different spacings between their peaks, and this causes a smearing out of the overall pattern. The second way in which the pattern can be degraded comes into play if the potential is different in different regions, in particular if it consists of two peaks, which are not the same. Then the path the molecule takes and its internal state can become entangled. If the internal states are sufficiently different, then they will reveal which path the molecule took, and there will be no interference pattern. Our model will allow us to study both of these mechanisms.

We calculate the cross section for our scattering in the Born approximation, which we must modify to take into account the internal (rotational) states of the molecule. This calculation is relatively standard, so we have relegated it to an appendix. We begin the next section with the expression for the cross section, and proceed to analyze its implications.

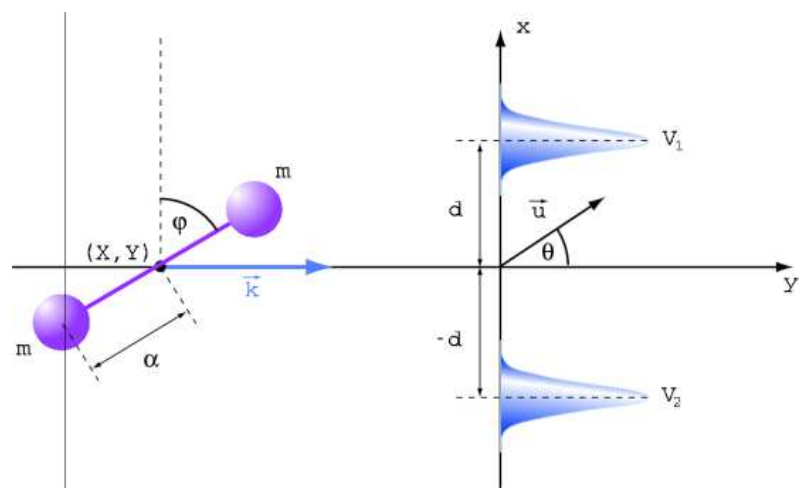

FIG. 1. Schematic representation of a scattering of a two-atom molecule on a potential composed of two (Gaussian) peaks. The two "atoms" of the molecule have a mass $m$, and each located a distance $\alpha$ from the center of mass of the molecule. The location of the center of mass is given by $(X, Y)$. The angle between the line connecting the two masses and the positive $x$ axis is $\phi$, and the angular momentum corresponding to the rotation of the molecule in the $x-y$ plane is $L$. The angle between the wave vector of the outgoing molecule and the positive $y$ axis is denoted by $\theta$, and $\hat{\mathbf{u}}$ is a unit vector pointing in the direction of the outgoing wave vector. 


\section{CROSS SECTION}

We begin by describing our system in more detail. The molecule consists of two "atoms", both of mass $m$, and each located a distance $\alpha$ from the center of mass of the molecule. The location of the center of mass is given by $(X, Y)$ and its momentum by $\left(P_{x}, P_{y}\right)$. The angle between the line connecting the two masses and the positive $x$ axis is $\phi$, and the angular momentum corresponding to the rotation of the molecule in the $x$ - $y$ plane is $L$. There is also a potential with which the particles interact, $V$. In Fig. 1 we present a schematic picture of the physical situation we consider. The Hamiltonian describing the system is

$$
H=\frac{1}{4 m}\left(P_{x}^{2}+P_{y}^{2}\right)+\frac{1}{2 I} L^{2}+V(X+\alpha \cos \phi, Y+\alpha \sin \phi)+V(X-\alpha \cos \phi, Y-\alpha \sin \phi),
$$

where $I=2 m \alpha^{2}$ is the moment of inertia of the molecule. We shall label the states of the system, $|\mathbf{k}, l\rangle$ by the wave vector of the center of mass, $\mathbf{k}$, and the quantum number of the rotational motion, $l$. The states are normalized so that

$$
\left\langle\mathbf{k}, l \mid \mathbf{k}^{\prime}, l^{\prime}\right\rangle=\delta^{2}\left(\mathbf{k}-\mathbf{k}^{\prime}\right) \delta_{l, l^{\prime}} .
$$

Now we consider the situation in which the molecule comes in from the negative $y$ direction with an initial state

$$
\left|\Psi_{i n}\right\rangle=\sum_{l=-\infty}^{\infty} \psi_{l}|\mathbf{k}=k \hat{\mathbf{y}}, l\rangle
$$

Let $\theta$ be the angle between the wave vector of the outgoing molecule (the wave vector after the scattering has take place) and the positive $y$ axis, and $\hat{\mathbf{u}}$ be a unit vector pointing in the direction of the outgoing wave vector. The cross section for the scattering is given by

$$
\sigma(\theta)=(2 \pi)^{3} \frac{4 m^{2}}{k} \sum_{l, l^{\prime}=-\infty}^{\infty} \Theta\left(\frac{k^{2}}{4 m}+\frac{l^{2}}{2 I}-\frac{\left(l^{\prime}\right)^{2}}{2 I}\right)\left|\psi_{l}\right|^{2}\left|\left\langle\kappa\left(k, l, ; l^{\prime}\right) \hat{\mathbf{u}}, l^{\prime}|V| k \hat{\mathbf{y}}, l\right\rangle\right|^{2},
$$

where $\Theta(x)=1$ if $x \geq 0$ and $\Theta(x)=0$, if $x<0$, and

$$
\kappa\left(k, l ; l^{\prime}\right)=2 \sqrt{m}\left(\frac{k^{2}}{4 m}+\frac{l^{2}}{2 I}-\frac{\left(l^{\prime}\right)^{2}}{2 I}\right)^{1 / 2} .
$$

This is to be compared with the cross section for the scattering, in two dimensions, of a particle of mass $\mathrm{M}$ and no internal structure from a potential $V(\mathbf{r})$, which is, in the Born approximation,

$$
\sigma(\theta)=\frac{M^{2}}{2 \pi k}\left|\int d^{2} r e^{-i k(\hat{\mathbf{u}}-\hat{\mathbf{y}}) \cdot \mathbf{r})} V(\mathbf{r})\right|^{2} .
$$

We now need to calculate the matrix element of the potential. First we shall assume that the potential is the sum of two terms, $V(\mathbf{r})=V_{1}(\mathbf{r})+V_{2}(\mathbf{r})$, where $V_{1}(\mathbf{r})$ is centered about the point $(d, 0)$ and $V_{2}(\mathbf{r})$ is centered about the point $(-d, 0)$. The distance $2 d$ plays the role of the distance between the slits in a two-slit interference experiment. The matrix element appearing in the cross section is then the sum of four terms, as both $V_{1}$ and $V_{2}$ are evaluated at the points $(X+\alpha \cos \phi, Y+\alpha \sin \phi)$ and $(X-\alpha \cos \phi, Y-\alpha \sin \phi)$, i.e. the coordinates of the two masses of the molecule. We define, for $j=1,2$,

$$
\tilde{V}_{j}\left(k_{x}, k_{y}\right)=\frac{1}{2 \pi} \int d x \int d y e^{-i\left(k_{x} x+k_{y} y\right)} V_{j}(x, y) .
$$

For the matrix element of $V_{j}(X+\alpha \cos \phi, Y+\alpha \sin \phi)$, which we shall denote by $V_{j}^{(+)}$, we then have

$$
\begin{aligned}
\left\langle\kappa \hat{\mathbf{u}}, l^{\prime}\left|V_{j}^{(+)}\right| k \hat{\mathbf{y}}, l\right\rangle & =\frac{1}{(2 \pi)^{3}} \int_{-\infty}^{\infty} d X \int_{-\infty}^{\infty} d Y \int_{0}^{2 \pi} d \phi e^{i(\kappa \hat{\mathbf{u}}-k \hat{\mathbf{y}}) \cdot \mathbf{R}} e^{i\left(l-l^{\prime}\right) \phi} V_{j}(X+\alpha \cos \phi, Y+\alpha \sin \phi) \\
& =\frac{1}{2 \pi} \tilde{V}_{j}(-\kappa \sin \theta, k-\kappa \cos \theta) \int_{0}^{2 \pi} d \phi e^{i\left(l-l^{\prime}\right) \phi} \exp [-i \kappa \alpha \sin \theta \cos \phi] \exp [i(k-\kappa \cos \theta) \alpha \sin \phi] \\
& =\frac{1}{(2 \pi)^{1 / 2}} e^{-i\left(l-l^{\prime}\right) \mu} J_{l-l^{\prime}}(\alpha|\kappa \hat{\mathbf{u}}-k \hat{\mathbf{y}}|) \tilde{V}_{j}(k \hat{\mathbf{y}}-\kappa \hat{\mathbf{u}})
\end{aligned}
$$


The angle $\mu$ satisfies

$$
\sin \mu=\frac{\kappa \sin \theta}{|\kappa \hat{\mathbf{u}}-k \hat{\mathbf{y}}|} ; \quad \cos \mu=\frac{\kappa \cos \theta-k}{|\kappa \hat{\mathbf{u}}-k \hat{\mathbf{y}}|}
$$

so that

$$
\mu=\tan ^{-1}\left[\frac{\kappa \sin \theta}{\kappa \cos \theta-k}\right]
$$

Note that the quadrant in which $\mu$ lies is specified by Eq. (2.9). The evaluation of the matrix element of $V_{j}(X-$ $\alpha \cos \phi, Y-\alpha \sin \phi)$, which we denote by $V_{j}^{(-)}$is similar, and we find

$$
\left\langle\kappa \hat{\mathbf{u}}, l^{\prime}\left|V_{j}^{(-)}\right| k \hat{\mathbf{y}}, l\right\rangle=\frac{1}{(2 \pi)^{1 / 2}} e^{-i\left(l-l^{\prime}\right) \mu}(-1)^{l-l^{\prime}} J_{l-l^{\prime}}(\alpha|\kappa \hat{\mathbf{u}}-k \hat{\mathbf{y}}|) \tilde{V}_{j}(k \hat{\mathbf{y}}-\kappa \hat{\mathbf{u}}) .
$$

Consequently, we have that

$$
\left\langle\kappa \hat{\mathbf{u}}, l^{\prime}|V| k \hat{\mathbf{y}}, l\right\rangle=\frac{1}{2 \pi} e^{-i\left(l-l^{\prime}\right) \mu}\left[1+(-1)^{l-l^{\prime}}\right] J_{l-l^{\prime}}(\alpha|\kappa \hat{\mathbf{u}}-k \hat{\mathbf{y}}|) \sum_{j=1}^{2} \tilde{V}_{j}(k \hat{\mathbf{y}}-\kappa \hat{\mathbf{u}}) .
$$

Let us begin by considering the case in which $V_{1}$ and $V_{2}$ are both Gaussians with the same width

$$
\begin{aligned}
& V_{1}(\mathbf{r})=V_{0} e^{-|\mathbf{r}-d \hat{\mathbf{x}}|^{2} / \Delta^{2}} \\
& V_{2}(\mathbf{r})=V_{0} e^{-|\mathbf{r}+d \hat{\mathbf{x}}|^{2} / \Delta^{2}}
\end{aligned}
$$

With this potential, the cross section becomes

$$
\begin{aligned}
\sigma(\theta) & =\frac{8 \pi m^{2} \Delta^{4} V_{0}^{2}}{k} \sum_{l, l^{\prime}=-\infty}^{\infty} \Theta\left(\frac{k^{2}}{4 m}+\frac{l^{2}}{2 I}-\frac{\left(l^{\prime}\right)^{2}}{2 I}\right)\left[1+(-1)^{l-l^{\prime}}\right]^{2} \\
& \times e^{-\Delta^{2}|\kappa \hat{\mathbf{u}}-k \hat{\mathbf{y}}|^{2} / 2} J_{l-l^{\prime}}^{2}(\alpha|\kappa \hat{\mathbf{u}}-k \hat{\mathbf{y}}|) \cos ^{2}(\kappa d \sin \theta)\left|\psi_{l}\right|^{2} .
\end{aligned}
$$

The presence of an interference pattern can be seen from the presence of the cosine term.

We shall examine this expression in a number of parameter regions. First, consider the case in which $\Delta \gg \alpha$, i.e. the potential varies slowly over distances of the order of the size of the molecule. We would not expect the fact that the molecule has internal degrees of freedom to play much of a role in this case, and this is, indeed what we find. Note that, because of the Gaussian factor, the cross section will be small unless $|\kappa \hat{\mathbf{u}}-k \hat{\mathbf{y}}| \Delta$ is of order one or smaller. We have that

$$
\begin{aligned}
|\kappa \hat{\mathbf{u}}-k \hat{\mathbf{y}}| \Delta & >|\kappa-k| \Delta \\
& >\left|\left[(k \Delta)^{2}+\left(\frac{\Delta}{\alpha}\right)^{2}\left[l^{2}-\left(l^{\prime}\right)^{2}\right]\right]^{1 / 2}-k \Delta\right| .
\end{aligned}
$$

From this expression we see that for $\Delta \gg \alpha$, we will have that $|\kappa \hat{\mathbf{u}}-k \hat{\mathbf{y}}| \Delta \gg 1$ unless $l=l^{\prime}$, and this implies that only the terms with $l=l^{\prime}$ will contribute to the sum in Eq. (2.14). In that case we have that $\kappa=k$, and

$$
\sigma(\theta)=\frac{32 \pi m^{2} \Delta^{4} V_{0}^{2}}{k} e^{-(k \Delta)^{2}|\hat{\mathbf{u}}-\hat{\mathbf{y}}|^{2} / 2} J_{0}^{2}(k \alpha|\hat{\mathbf{u}}-\hat{\mathbf{y}}|) \cos ^{2}(k d \sin \theta) .
$$

Note that, as expected, all of the dependence on the internal states is gone. If, in addition, the molecule is much smaller than the wavelength corresponding to its center-of-mass motion, then the Bessel function can be replaced by 1 , and all vestige of internal structure disappears. In fact, in this limit the scattering cross section becomes the same as that of a particle with no internal structure and a mass of $2 m$ scattering from a potential $2\left(V_{1}(\mathbf{r})+V_{2}(\mathbf{r})\right)($ see Eq. $(2.6))$.

In the experiments with fullerenes, the ratio of the slit separation to the de Broglie wavelength of the molecules was of the order of $10^{4}$, and the scattering only at angles near the forward direction was observed. Let us see what our toy model predicts for a situation similar to this one. We shall assume that $k d \sim 10^{4}$, $\alpha<d$ but the two are of 
the same order of magnitude, and, for simplicity, that our molecule starts in the $l=0$ state. In addition, we shall concentrate our attention on angles near the forward direction, in particular, $\theta \sim 10^{-4}$. We now have that

$$
\kappa=k\left[1-\frac{\left(l^{\prime}\right)^{2}}{(k \alpha)^{2}}\right]^{1 / 2}
$$

so that the only angular momentum states that can be excited are the ones satisfying $k \alpha \geq l^{\prime}$. Since $k \alpha \sim 10^{4}$, it seems that many internal states can be excited, and that this could have an effect on the interference pattern. In particular, the interference pattern is given by the $\cos (\kappa d \sin \theta)$ factor, so that if many values of $l^{\prime}$, and hence many values of $\kappa$, are allowed, then the interference pattern could be smeared out.

This does not happen in this situation. As was noted earlier, in order for the Gaussian factor not to cut everything off, we must have $|k-\kappa|$ of order one or less, that is

$$
k \Delta\left\{1-\left[1-\frac{\left(l^{\prime}\right)^{2}}{(k \alpha)^{2}}\right]^{1 / 2}\right\}<1
$$
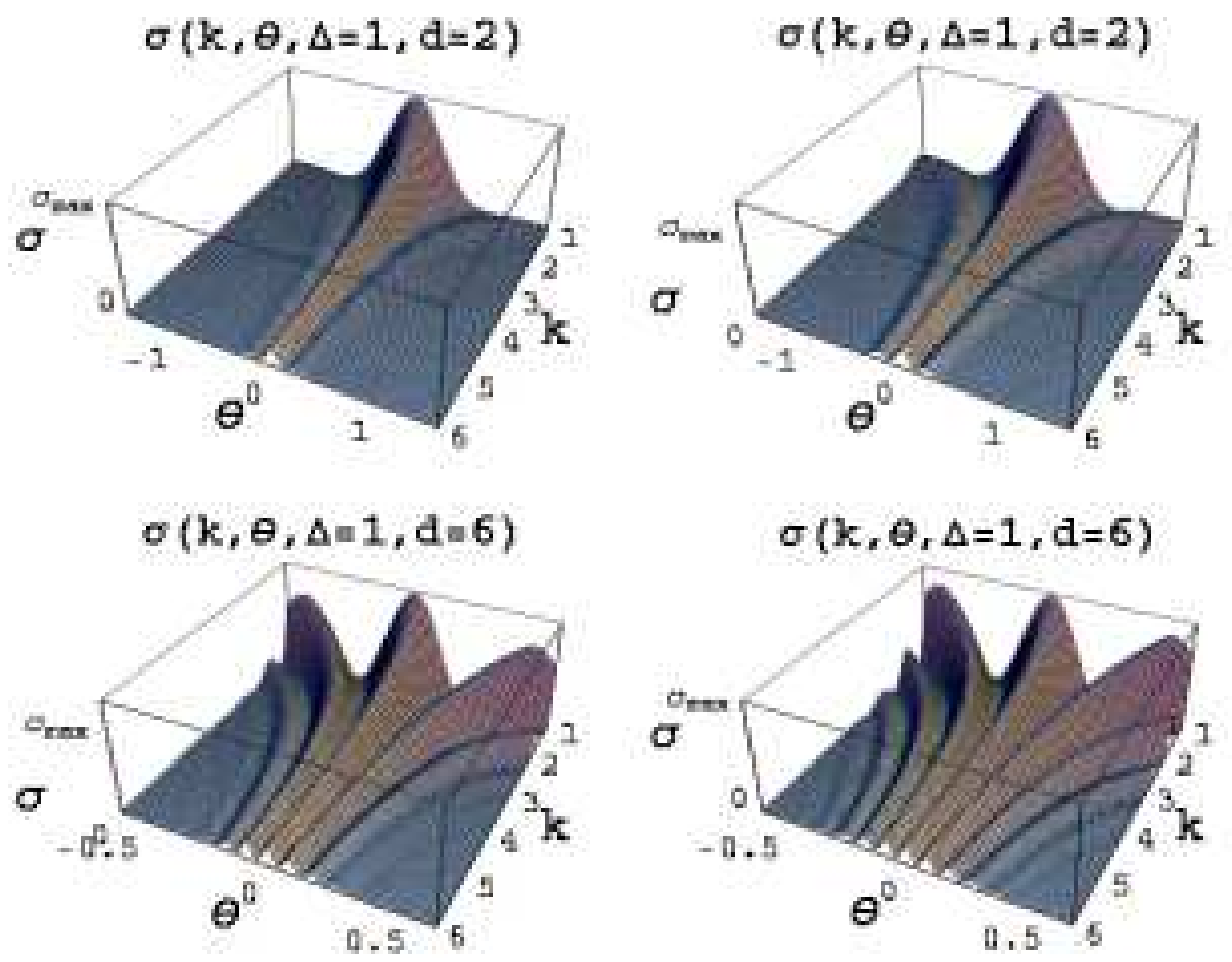

FIG. 2. We plot the cross section $\sigma(\theta)$ as a function of $\theta$ and the momentum $k$. We compare two cases: a molecule with (left column) and without (right column) internal degrees of freedom. From the figure it is obvious that the internal structure of the molecule causes a suppression of the interference pattern. The cross section $\sigma$ in the presence of the internal structure of the molecule is represented by Eq.(2.14) where we assume that the incident molecule is in the state with $l=0$. The cross section corresponding to the case with a molecule that does not have an internal structure is given by Eq.(2.21). Otherwise the molecules are considered to be the same, i.e. we assume units such that $m=1$ and $\hbar=1$. The two Gaussian peaks are characterized by $V_{0}=1$ and $\Delta=1$. The distance between each scattering peak and the origin of the coordinate system is $d=2$ (the upper row) and $d=6$ (the lower row). We observe that with larger $d$ the frequency of interference oscillations is larger. The suppression of oscillations due to the presence of internal molecular states is clearly seen in both cases, i.e. $d=2$ and $d=6$.

Let us assume that $\Delta<d$, but that $k \Delta \gg 1$. Then in order to satisfy the above condition, we must have $l^{\prime} /(k \alpha) \ll 1$, and, in particular, $l^{\prime} /(k \alpha)<1 / \sqrt{k \Delta}$. This implies that the values of $\kappa$ that contribute to the sum in the 
expression for the cross section, lie in the range between $k$ and $k-(1 / \Delta)$, that is, all of these values are very close to $k$. Making the approximation that $\kappa=k$, we find that

$$
|\kappa \hat{\mathbf{u}}-k \hat{\mathbf{y}}|=\sqrt{2} k(1-\cos \theta)^{1 / 2},
$$

and

$$
\begin{aligned}
\sigma(\theta) & =\frac{8 \pi m^{2} \Delta^{4} V_{0}^{2}}{k} \sum_{l^{\prime}=-\infty}^{\infty} \Theta\left(k^{2}-\frac{\left(l^{\prime}\right)^{2}}{\alpha^{2}}\right)\left[1+(-1)^{l^{\prime}}\right]^{2} \\
& \times e^{-(k \Delta)^{2}(1-\cos \theta) / 2} J_{l^{\prime}}^{2}\left(\sqrt{2} \alpha k(1-\cos \theta)^{1 / 2}\right) \cos ^{2}(k d \sin \theta) .
\end{aligned}
$$

In the range $\theta \sim(1 / k d) \sim 10^{-4}$, we find that the quantities $k \Delta(1-\cos \theta)^{1 / 2}, k \alpha(1-\cos \theta)^{1 / 2}$, and $k d \sin \theta$ are all of order one, and the cross section exhibits strong interference fringes.

In our examples so far, the internal states have not influenced the interference pattern. One regime in which they do is when $k \Delta, k \alpha$, and $k d$ are roughly of order one. In Fig. 2 we present the cross section $\sigma(\theta)$ as a function of $\theta$ and the momentum $k$ for parameters in this range. We compare two cases: Firstly, we consider the case when the molecule has an internal structure and the cross section $\sigma(\theta)$ is described by Eq. (2.14). Secondly, we analyze the situation when the molecule has no internal structure. In all cases when we are comparing scattering with and without internal structure, the molecule without internal structure is taken to have a mass of $2 m$, and the potential acting on it is twice that of the potential for the molecule with internal structure (this is to compensate for the fact that for the molecule with internal structure, the potential acts on each particle). For this case using the general expression given by Eq. (2.6) we can derive the cross section for the scattering potential with two Gaussian peaks in the form:

$$
\sigma(\theta)=\frac{32 \pi m^{2} \Delta^{4} V_{0}^{2}}{k} \exp \left[-\Delta^{2} k^{2}(1-\cos \theta)\right] \cos ^{2}(k d \sin \theta) .
$$

From Fig. 2 it is obvious that the internal structure of the molecule causes a suppression of the interference pattern. In the figure we use units and values of the parameters involved in the expressions for the cross section such that $k \Delta$, $k \alpha$ and $k d$ are not too large (of the order between 1 and 10 in dimensionless units used in the figure).

The actual experiments use a grating instead of two slits, and this it is straightforward to incorporate this into our model. Suppose the the potential consists of $2 N+1$ peaks centered on the $x$ axis, so that

$$
V(\mathbf{r})=\sum_{n=-N}^{N} v(\mathbf{r}-n d \hat{\mathbf{x}}),
$$

where $v(\mathbf{r})$ is the potential for one of the peaks. We then find that

$$
\tilde{V}(\mathbf{k})=\sum_{n=-N}^{N} e^{-i k_{x} n d} \tilde{v}(\mathbf{k})=\frac{\sin \left[k_{x} d(2 N+1) / 2\right]}{\sin \left(k_{x} d / 2\right)} \tilde{v}(\mathbf{k}) .
$$

In the case that the peaks are Gaussian,

$$
v(\mathbf{r})=V_{0} e^{-|\mathbf{r}|^{2} / \Delta^{2}}
$$

the cross section becomes

$$
\begin{aligned}
\sigma(\theta) & =\frac{2 \pi m^{2} \Delta^{4} V_{0}^{2}}{k} \sum_{l^{\prime}=-\infty}^{\infty} \Theta\left(k^{2}-\frac{\left(l^{\prime}\right)^{2}}{\alpha^{2}}\right)\left[1+(-1)^{l^{\prime}}\right]^{2} \\
& \times e^{-(\Delta)^{2}|\kappa \hat{\mathbf{u}}-k \hat{\mathbf{y}}|^{2} / 2} J_{l^{\prime}}^{2}(\sqrt{2} \alpha|\kappa \hat{\mathbf{u}}-k \hat{\mathbf{y}}|) \frac{\left.\sin ^{2}[\kappa d(2 N+1) \sin \theta) / 2\right]}{\sin ^{2}[(\kappa d \sin \theta) / 2]} .
\end{aligned}
$$

If we are in the regime in which the experiments were done, i.e. $k d \sim 10^{4}$ and $\alpha$ and $\Delta$ both less than $d$ but of similar order of magnitude, the same considerations as those in the derivation of Eq. (2.20) apply, and we find for the cross section

$$
\begin{aligned}
\sigma(\theta) & =\frac{2 \pi m^{2} \Delta^{4} V_{0}^{2}}{k} \sum_{l^{\prime}=-\infty}^{\infty} \Theta\left(k^{2}-\frac{\left(l^{\prime}\right)^{2}}{\alpha^{2}}\right)\left[1+(-1)^{l^{\prime}}\right]^{2} \\
& \times e^{-(k \Delta)^{2}(1-\cos \theta) / 2} J_{l^{\prime}}^{2}\left(\sqrt{2} \alpha k(1-\cos \theta)^{1 / 2}\right) \frac{\sin ^{2}[(k d(2 N+1) \sin \theta) / 2]}{\sin ^{2}[(k d \sin \theta) / 2]}
\end{aligned}
$$


We note that near $\theta=0$ the spacing between peaks is roughly $2 \pi /[k d(2 N+1)]$ (the first peak is at $\theta=0$, the second, which is not as high, is between $2 \pi /[k d(2 N+1)]$ and $4 \pi /[k d(2 N+1)])$. In the experiment, a molecule passes through about 100 slits (this is the beam width divided by the slit spacing), which gives a value of $N$ of around 50 . This implies an angular spacing between peaks in the pattern near $\theta=0$ of $10^{-5}$ to $10^{-6}$, which agrees with what was seen.
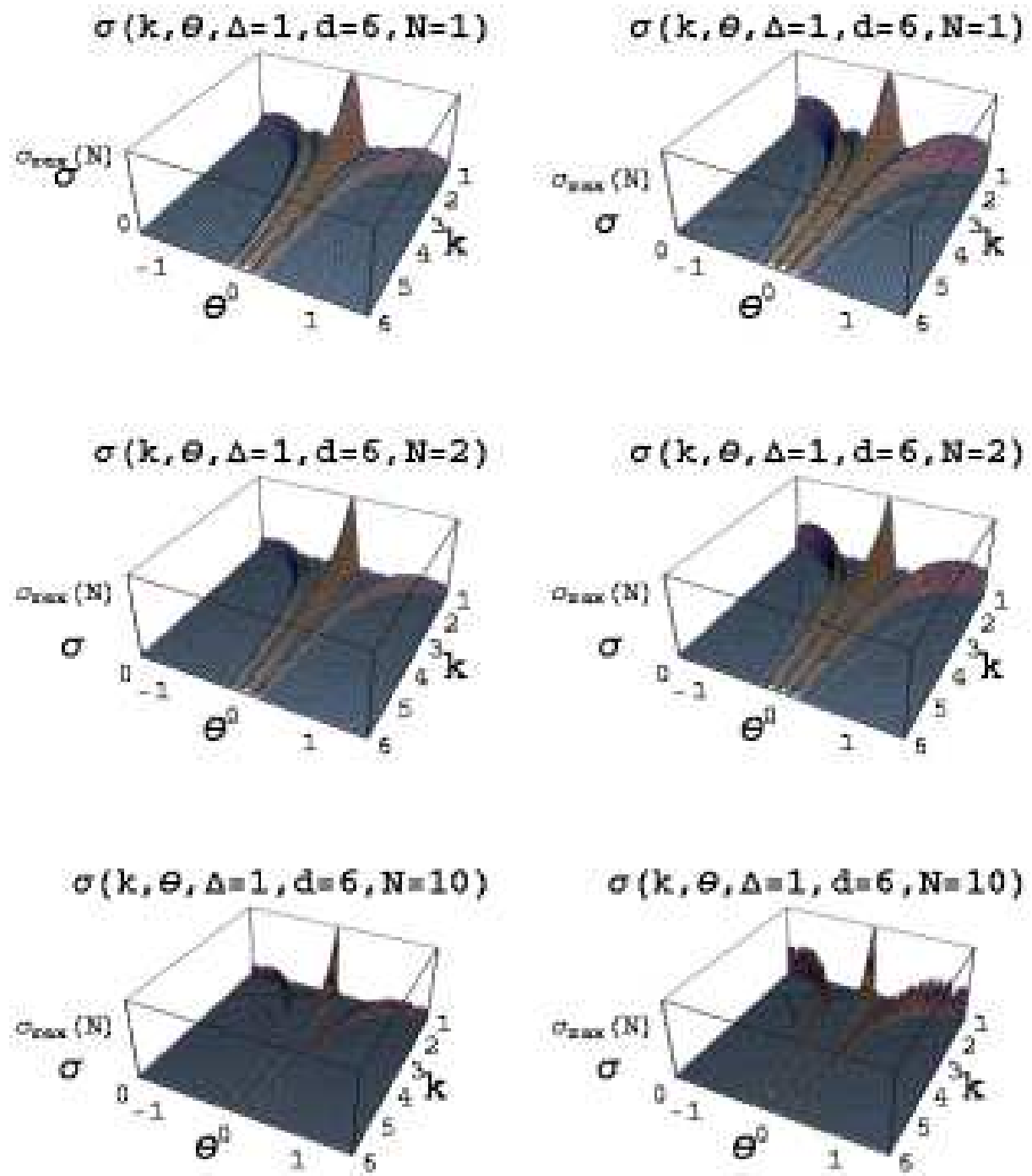

FIG. 3. We plot the cross section $\sigma(\theta)$ of the molecule that is scattered by a grating modelled as a potential with $2 N+1$ Gaussian peaks. We present the cross section as a function of $\theta$ and the momentum $k$ for different values of $N$. We compare two cases: We assume the molecule with (left column) and without (right column) internal degrees of freedom. From the figure it is obvious that the internal structure of the molecule causes a suppression of the interference pattern. The cross section $\sigma$ in the presence of the internal structure of the molecule is represented by Eq.(2.25) where we assume that the incident molecule is in the state with $l=0$. The cross section corresponding to the case with a molecule that does not have an internal structure is given by Eq.(2.27). Otherwise the molecules are considered to be the same and we assume units such that $m=1$ and $\hbar=1$. The Gaussian peaks are characterized by $V_{0}=1$ and $\Delta=1$. The distance between adjacent scattering peaks is $d=6$. We consider three different types of gratings with $N=1$ (i.e. scattering on 3 Gaussian peaks - see the first row), with $N=2$ (i.e. scattering on 5 Gaussian peaks - see the second row); and with $N=10$ (i.e. scattering on 21 Gaussian peaks - see the third row). The maximal values of the cross section that are achieved from small $k$ and $\theta=0$ are different for different values of $N$. Particularly, these values are proportional to $N^{2}$. In any case, we see a suppression of the quantum interference patterns due to the presence of internal molecular states. 
In order to investigate the role of internal states for the case of a grating potential, we again go the the regime in which $k \Delta, k \alpha$, and $k d$ are roughly of order one. The expression for the cross section of a molecule with no internal structure scattering off of a potential with $2 N+1$ identical peaks can be found by using the general expression given by Eq. (2.6) and is given by

$$
\sigma(\theta)=\frac{32 \pi m^{2} \Delta^{4} V_{0}^{2}}{k} \exp \left[-\Delta^{2} k^{2}(1-\cos \theta)\right] \frac{\sin ^{2}[(k d(2 N+1) \sin \theta) / 2]}{\sin ^{2}[(k d \sin \theta) / 2]} .
$$

We plot the cross sections $\sigma(\theta)$ given by Eqs. (2.25) and (2.27) in Fig. 3 as a function of $\theta$ and $k$ for different values of $N$. From the figure we can again conclude that the presence of the internal structure of the molecule leads to a suppression of the interference pattern.

\section{DIFFERENT PEAKS}

So far we have considered only the case in which both of the peaks in the potential are the same. We shall now consider the case in which they are not. This will give rise to another mechanism that can decrease the visibility of the interference pattern. The different peaks will give rise to different excitations of the internal states, and the interference pattern will be proportional to the overlap between these internal states. The overlap is related to the information about the path the molecule followed through the potential. If the overlap is zero, then by looking at the internal state of the molecule, we can determine from which peak it scattered, then there will be no interference pattern. If the overlap is greater than zero, then there is partial information about the path, and the visibility of the interference pattern is correspondingly reduced.

We shall now assume that the potential is the sum of two terms, $V(\mathbf{r})=V_{1}(\mathbf{r}-d \hat{\mathbf{x}})+V_{2}(\mathbf{r}+d \hat{\mathbf{x}})$, where

$$
\begin{aligned}
& V_{1}(\mathbf{r})=V_{0}\left(1-\frac{r^{2}}{\Delta^{2}}\right) e^{-(r / \Delta)^{2}} \\
& V_{2}(\mathbf{r})=V_{0} e^{-(r / \Delta)^{2}} .
\end{aligned}
$$

The Fourier transforms of these potentials are given by

$$
\begin{array}{r}
\tilde{V}_{1}(\mathbf{k})=\frac{1}{8} V_{0} k^{2} \Delta^{4} e^{-(k \Delta)^{2} / 4} ; \\
\tilde{V}_{2}(\mathbf{k})=\frac{1}{2} V_{0} \Delta^{2} e^{-(k \Delta)^{2} / 4},
\end{array}
$$

so that the matrix element of the total potential is

$$
\begin{aligned}
\left\langle\kappa \hat{\mathbf{u}}, l^{\prime}|V| k \hat{\mathbf{y}}, l\right\rangle & =\frac{1}{2 \pi} e^{-i\left(l-l^{\prime}\right)}\left[1+(-1)^{l-l^{\prime}}\right] J_{l-l^{\prime}}(\alpha|\kappa \hat{\mathbf{u}}-k \hat{\mathbf{y}}|) \\
& \times \frac{1}{2} V_{0} \Delta^{2} e^{-|\kappa \hat{\mathbf{u}}-k \hat{\mathbf{y}}|^{2} \Delta^{2} / 4}\left[\frac{\Delta^{2}}{4}|\kappa \hat{\mathbf{u}}-k \hat{\mathbf{y}}|^{2} e^{i \kappa d \sin \theta}+e^{i \kappa d \sin \theta}\right] .
\end{aligned}
$$

Now consider a particular example. As usual, we shall assume that the molecule is initially in the $l=0$ state. We shall also assume that $3>k \alpha \geq 1$, which means that the there are three terms in the $l^{\prime}$ sum (the $l^{\prime}= \pm 1$ terms vanish). Let us now examine the terms corresponding to $V_{1}$ and $V_{2}$ in the above matrix element. Define the magnitude of the difference between the outgoing and incoming momenta to be $q=|\mathbf{q}|$ where $\mathbf{q}=\kappa \hat{\mathbf{u}}-k \hat{\mathbf{y}}$. The $V_{1}$ contribution at $l^{\prime}=0$ is proportional to $J_{0}(\alpha q) q^{2} \exp \left[-(q \Delta / 2)^{2}\right]$ while the $V_{2}$ contribution is proportional to $J_{0}(\alpha q) \exp \left[-(q \Delta / 2)^{2}\right]$. The function $q^{2} \exp \left[-(q \Delta / 2)^{2}\right]$ has a maximum at $q=2 / \Delta$. If $\alpha$ and $\Delta$ are chosen so that this maximum occurs at a zero of $J_{0}(\alpha q)$, then the contribution of $V_{1}$ to the scattering at $l^{\prime}=0$ will be greatly suppressed. What will happen then is that $V_{1}$ will scatter the molecule primarily into the $l^{\prime}= \pm 2$ states and $V_{2}$ will scatter it primarily into the $l^{\prime}=0$ state. The result will be a suppression of the interference pattern.

Let us make this more specific. First, the cross-section for this choice of potential and the molecule initially in the $l=0$ state is given by

$$
\sigma(\theta)=\frac{2 \pi m^{2} V_{0}^{2} \Delta^{4}}{k} \sum_{l^{\prime}=-\infty}^{\infty}\left[1+(-1)^{l^{\prime}}\right]^{2} J_{l^{\prime}}^{2}(\alpha q) e^{-(q \Delta)^{2} / 2}\left[1+\frac{(q \Delta)^{4}}{16}+\frac{(q \Delta)^{2}}{2} \cos (2 \kappa d \sin \theta)\right] .
$$


For the same choice of the potential but for the molecule without an internal structure we find the cross section using Eq. (2.6) in a form

$$
\sigma(\theta)=\frac{8 \pi m^{2} V_{0}^{2} \Delta^{4}}{k} e^{-(q \Delta)^{2} / 2}\left[1+\frac{(q \Delta)^{4}}{16}+\frac{(q \Delta)^{2}}{2} \cos (2 \kappa d \sin \theta)\right]
$$

In Fig. 4 we plot the cross sections $\sigma(\theta)$ given by Eqs. (3.4) and (3.5). We consider the case when $k \alpha=5 / 2, k \Delta=3 / 2$, and $k d=4$. In this case we see that the interference pattern is almost completely suppressed by decoherence due to the internal states of the molecule.

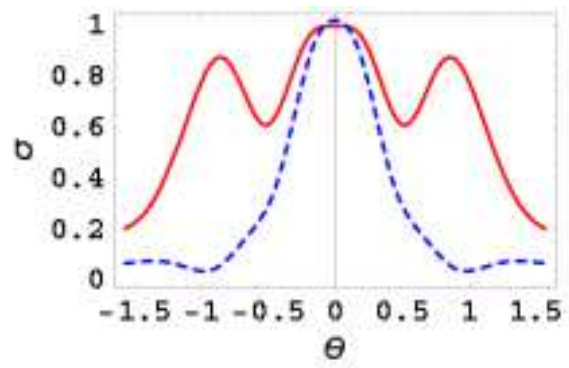

FIG. 4. We plot the cross section $\sigma(\theta)$ of the molecule that is scattered by a potential composed of two non-identical peaks. The potential is described by Eq. (3.1). We consider two situations: When the molecule has an internal structure (dashed line) given by Eq. (3.4) and when the molecule does not have an internal structure (solid line) given by Eq. (3.5). When internal states of the molecule are present, then depending on which path the molecule follows through the potential, that is which peak it scatters from, different (distinguishable) internal states of the molecule are excited. Consequently, in principle the path can be determined, and the interference is suppressed (this situation situation is very similar to that discussed in papers on "which-way information" in double-slit experiments - see e.g. Refs. [18,19]). Here the cross section is given by Eq. (3.4). We consider parameters such that $k \alpha=5 / 2, k \Delta=3 / 2$, and $k d=4$, and we obtain the cross section given by the dashed line. When the molecule has no internal structure, the path cannot be determined and the interference pattern is preserved (solid line). The cross section $\sigma(\theta)$ in this case is given by Eq. (3.5) and for the same values of parameters as before (i.e. $k \alpha=5 / 2$, $k \Delta=3 / 2$, and $k d=4)$.

\section{CONCLUSION}

The coupling of internal and translational degrees of freedom of an object can lead to the degradation of an interference pattern produced by the scattering of the object from a potential. This is a result of the entanglement between the internal and translational degrees of freedom of the object produced by the potential. We have studied two different forms this entanglement can take. In the first different outgoing wave vectors become entangled with different internal states producing an interference pattern made up of parts with different periodicities, which leads to a smearing the overall pattern. The second results from the entanglement of the internal states and different paths the molecule can follow through the potential.

Even though our model was very simple, it is possible to draw some conclusions from the results based on it, and to put forward some conjectures. In order for internal states to be excited, they must be of sufficiently low energy. If the molecule is of size $\mathrm{L}$ and mass $\mathrm{M}$, the lowest rotational mode will have an energy of order $1 / M L^{2}$. We would expect the mass of the molecule to scale roughly as its volume, which means that $M \sim L^{3}$, so that the energy of a low-lying rotational state is proportional to $1 / L^{5}$. There will also be vibrational modes. The energy of the low-lying acoustic phonon modes will be proportional to $1 / L$. In both cases, it is clear that the larger the object, the more low energy internal states it will have.

In our simple model, the coupling between the translational motion and the internal state with angular momentum $l$ (if the molecule was initially in the zero angular momentum state) is determined by the product $J_{l}(\alpha q) \tilde{V}(\mathbf{q})$. Therefore, if the Fourier transform of the potential is significant in the region near $l / \alpha$, where $J_{l}(\alpha q)$ is largest in absolute value, then there will be a a good chance of exciting the $l$ th rotational state. This implies that in order to excite internal states, the potential must vary on a scale of the order of the size of the object or smaller. Clearly this restriction is much less stringent for a large object than a small one.

These considerations suggest that it is easier to excite internal states in a large object than in a small one. There are more low lying states, and for a given potential, the probability of exciting one of these states is greater for the larger object, because the conditions for doing so are less restrictive. This further suggests that it is more likely for 
the translational motion of a large object to become entangled with its internal states than would be the case of a smaller object.

This gives us a possible mechanism for the emergence of classical behavior for an object with internal structure moving in a potential. If we consider the path-integral description of its dynamics, each of the paths it can follow will correspond to different internal excitations. Therefore, these paths will decohere, and instead of a coherent superposition of paths, we will have an incoherent one. In addition, the classical path will be the most probable, so that the object will simply follow this path through the potential.

There are clearly many gaps to be filled in before we can claim that this picture is correct. The calculations in this paper are a first step. More sophisticated models and treatments are called for. However, even this very simple model shows that internal states can act as a reservoir and cause different states of translational motion to decohere.

\section{ACKNOWLEDGMENTS}

This research was supported by the National Science Foundation under grant PHY 0139692. In addition this work was supported in part by the European Union projects QGATES and CONQUEST, by the Slovak Academy of Sciences via the project CE-PI and by the project APVT-99-012304. We thank Klaus Hornberger and Markus Arndt for very helpful correspondence.

\section{APPENDIX: DERIVATION OF THE CROSS SECTION}

Here we calculate the cross section for the scattering of our molecule from a potential, to first order in the potential. If the initial state of the molecule is $|\mathbf{k}, l\rangle$, and we want to find the amplitude to scatter into the state $\left|\mathbf{k}^{\prime}, l^{\prime}\right\rangle$. To lowest order in the potential, the S-matrix element for this process is

$$
\left\langle\mathbf{k}^{\prime}, l^{\prime}|S| \mathbf{k}, l\right\rangle=\delta^{(2)}\left(\mathbf{k}^{\prime}-\mathbf{k}\right) \delta_{l, l^{\prime}}-2 \pi i \delta\left(\frac{\left(k^{\prime}\right)^{2}}{4 m}+\frac{\left(l^{\prime}\right)^{2}}{2 I}-\frac{k^{2}}{4 m}-\frac{l^{2}}{2 I}\right)\left\langle\mathbf{k}^{\prime}, l^{\prime}|V| \mathbf{k}, l\right\rangle .
$$

In order to find the scattering amplitude for a more general initial state,

$$
\left|\Psi_{i n}\right\rangle=\sum_{l=-\infty}^{\infty} \int d^{2} k \Psi_{i n}(\mathbf{k}, l)|\mathbf{k}, l\rangle
$$

we simply multiply both sides of Eq. (A1) by $\Psi_{i n}(\mathbf{k}, l)$, integrate over $\mathbf{k}$, and sum over $l$.

In order to find the cross section, we shall follow the treatment in Reference [17]. The scattered wave function, $\Phi_{s}(\mathbf{k}, l)$ is given by

$$
\Phi_{s}\left(\mathbf{k}^{\prime}, l^{\prime}\right)=-2 \pi i \sum_{l=-\infty}^{\infty} \int d^{2} k \delta\left(\frac{\left(k^{\prime}\right)^{2}}{4 m}+\frac{\left(l^{\prime}\right)^{2}}{2 I}-\frac{k^{2}}{4 m}-\frac{l^{2}}{2 I}\right)\left\langle\mathbf{k}^{\prime}, l^{\prime}|V| \mathbf{k}, l\right\rangle \Psi_{i n}(\mathbf{k}, l) .
$$

If $\hat{\mathbf{u}}$ is a unit vector in the $\mathbf{k}^{\prime}$ direction, then the probability of the particle scattering in the $\hat{\mathbf{u}}$ direction, $P(\hat{\mathbf{u}})$ is

$$
P(\hat{\mathbf{u}})=\sum_{l^{\prime}=-\infty}^{\infty} \int d k^{\prime} k^{\prime}\left|\Phi_{s}\left(k^{\prime} \hat{\mathbf{u}}, l^{\prime}\right)\right|^{2}
$$

Substituting Eq. (A3) into the above equation and evaluating the $k^{\prime}$ integral gives us

$$
\begin{aligned}
P(\hat{\mathbf{u}}) & =8 \pi^{2} m \sum_{l^{\prime}=-\infty}^{\infty} \sum_{l_{1}, l_{2}=-\infty}^{\infty} \int d^{2} k_{1} \int d^{2} k_{2} \delta\left(\frac{k_{1}^{2}}{4 m}+\frac{l_{1}^{2}}{2 I}-\frac{k_{2}^{2}}{4 m}-\frac{l_{2}^{2}}{2 I}\right) \\
& \times \Theta\left(\frac{k_{1}^{2}}{4 m}+\frac{l_{1}^{2}}{2 I}-\frac{\left(l^{\prime}\right)^{2}}{2 I}\right)\left\langle\mathbf{k}_{2}, l_{2}|V| \kappa\left(k_{1}, l_{1} ; l^{\prime}\right) \hat{\mathbf{u}}, l^{\prime}\right\rangle\left\langle\kappa\left(k_{1}, l_{1} ; l^{\prime}\right) \hat{\mathbf{u}}, l^{\prime}|V| \mathbf{k}_{1}, l_{1}\right\rangle \Psi_{i n}^{*}\left(\mathbf{k}_{2}, l_{2}\right) \Psi_{i n}\left(\mathbf{k}_{1}, l_{1}\right),
\end{aligned}
$$

where $\kappa$ is defined in Eq. (2.5).

Now let us consider an incoming beam of particles that scatter off of the potential. The particles are in wave packets $\Phi_{\text {in }}(\mathbf{k}, l)=\phi_{\text {in }}(\mathbf{k}) \psi_{l}$, where $\phi_{\text {in }}$ is highly localized about $\mathbf{k}=k \hat{\mathbf{y}}$. The incoming beam is of width $2 R$, so that the 
center of the wave packets can be displaced in the $x$ direction anywhere between $-R \leq x \leq R$. Therefore, we consider incoming wave functions of the form

$$
\Psi_{i n}(\mathbf{k}, l)=e^{-i k_{x} x} \Phi_{i n}(\mathbf{k}, l),
$$

where $-R \leq x \leq R$. Consequently, we replace $\Psi_{i n}(\mathbf{k}, l)$ in Eq. (A5) by the above expression, and then average the result over $x$, i.e. we find $(1 / 2 R) \int_{-R}^{R} d x P(\hat{\mathbf{u}})$. Now $2 R P(\hat{\mathbf{u}})$ is just the length of the part of the incoming beam that scatters in the direction $\hat{\mathbf{u}}$, and this is just the cross section, $\sigma(\hat{\mathbf{u}})$. We, therefore, have

$$
\sigma(\hat{\mathbf{u}})=\int_{-R}^{R} d x P(\hat{\mathbf{u}})
$$

Assuming the scattering center is much smaller than the beam width, we can take the limit $R \rightarrow \infty$ in this equation, which introduces a $2 \pi \delta\left(k_{1 x}-k_{2 x}\right)$ into the integrals appearing in Eq. (A5). The result is

$$
\sigma(\hat{\mathbf{u}})=16 \pi^{3} m \sum_{l^{\prime}=-\infty}^{\infty} \sum_{l_{1}, l_{2}=-\infty}^{\infty} \int d^{2} k_{1} \int d^{2} k_{2} \delta\left(\frac{k_{1}^{2}}{4 m}+\frac{l_{1}^{2}}{2 I}-\frac{k_{2}^{2}}{4 m}-\frac{l_{2}^{2}}{2 I}\right) \delta\left(k_{1 x}-k_{2 x}\right) F\left(\mathbf{k}_{1}, \mathbf{k}_{2} ; l^{\prime}, l_{1}, l_{2}\right),
$$

where

$$
\begin{aligned}
F\left(\mathbf{k}_{1}, \mathbf{k}_{2} ; l^{\prime}, l_{1}, l_{2}\right) & =\Theta\left(\frac{k_{1}^{2}}{4 m}+\frac{l_{1}^{2}}{2 I}-\frac{\left(l^{\prime}\right)^{2}}{2 I}\right)\left\langle\mathbf{k}_{2}, l_{2}|V| \kappa\left(k_{1}, l_{1} ; l^{\prime}\right) \hat{\mathbf{u}}, l^{\prime}\right\rangle \\
& \times\left\langle\kappa\left(k_{1}, l_{1} ; l^{\prime}\right) \hat{\mathbf{u}}, l^{\prime}|V| \mathbf{k}_{1}, l_{1}\right\rangle \phi_{i n}^{*}\left(\mathbf{k}_{2}\right) \phi_{i n}\left(\mathbf{k}_{1}\right) \psi_{l_{2}}^{*} \psi_{l_{1}} .
\end{aligned}
$$

Our remaining task is to evaluate the integrals. Let us first do the $\mathbf{k}_{2}$ integral. The integral over $k_{2 x}$ simply sets $k_{2 x}=k_{1 x}$, and then the $k_{2 y}$ integral sets $k_{2 y}= \pm \kappa\left(k_{1 y}, l_{1} ; l_{2}\right)$. The result is

$$
\sigma(\hat{\mathbf{u}})=16 \pi^{3} m \sum_{l^{\prime}=-\infty}^{\infty} \sum_{l_{1}, l_{2}=-\infty}^{\infty} \int d^{2} k_{1} \frac{2 m}{\kappa\left(k_{1 y}, l_{1} ; l_{2}\right)} \sum_{ \pm} F\left(k_{1 x}, k_{1 y}, k_{1 x}, \pm \kappa\left(k_{1 y}, l_{1} ; l_{2}\right) ; l^{\prime}, l_{1}, l_{2}\right) .
$$

Now suppose that $\phi_{i n}(\mathbf{k})=\phi_{i n x}\left(k_{x}\right) \phi_{i n y}\left(k_{y}\right)$, where $\phi_{i n x}$ is localized about zero and $\phi_{i n y}$ is localized about $k$. Then the effect of doing the $k_{1 x}$ integral is simply to set $k_{1 x}=0$ in the integrand. The result is

$$
\begin{aligned}
\sigma(\hat{\mathbf{u}})= & 16 \pi^{3} m \sum_{l^{\prime}=-\infty}^{\infty} \sum_{l_{1}, l_{2}=-\infty}^{\infty} \int d k_{1 y} \frac{2 m}{\kappa\left(k_{1 y}, l_{1} ; l_{2}\right)} \Theta\left(\frac{k_{1 y}^{2}}{4 m}+\frac{l_{1}^{2}}{2 I}-\frac{\left(l^{\prime}\right)^{2}}{2 I}\right) \\
& \sum_{ \pm}\left\langle \pm \kappa\left(k_{1 y}, l_{1} ; l_{2}\right) \hat{\mathbf{y}}, l_{2}|V| \kappa\left(k_{1 y}, l_{1} ; l^{\prime}\right) \hat{\mathbf{u}}, l^{\prime}\right\rangle\left\langle\kappa\left(k_{1 y}, l_{1} ; l^{\prime}\right) \hat{\mathbf{u}}, l^{\prime}|V| k_{1 y} \hat{\mathbf{y}}, l_{1}\right\rangle \phi_{i n y}^{*}\left( \pm \kappa\left(k_{1 y}, l_{1} ; l_{2}\right)\right) \phi_{i n y}\left(k_{1 y}\right) \psi_{l_{2}}^{*} \psi_{l_{1}}
\end{aligned}
$$

We only get a substantial contribution to the remaining integral when $k_{1 y} \sim k$ and $\kappa\left(k_{1 y}, l_{1} ; l_{2}\right) \sim k$. Note that this implies that only the + in the sum over + and - contributes. These two conditions imply that $\kappa\left(k, l_{1} ; l_{2}\right) \sim k$, which further implies that $l_{1}=l_{2}$. Doing the $k_{1 y}$ integral, then, has the effect of setting $k_{1 y}=k$ and $l_{1}=l_{2}$ in the integrand, and the result is Eq. (2.4).

[1] I. Estermann and O. Stern, Z. Phys. 61, 95 (1930).

[2] C. Bordé, N. Courtier, F. D. Burck, A. Goncharov, and M. Gorlicki, Phys. Lett. A 188, 187 (1994).

[3] C. Lisdat, M. Frank, H. Knockel, M. -L. Almazor, and E. Tiemann, Eur. Phys. J. D 12, 235 (2000).

[4] M. S. Chapman, C. R. Ekstrom, T. D. Hammond, R. A. Rubenstein, J. Schmiedmayer, S. Wehringer, and D. E. Pritchard, Phys. Rev. Lett. 74, 4783 (1995).

[5] See comment by J. Clauser in aAtom Interferometry, edited by P. R. Berman (Academic, New York, 1997).

[6] R. E. Grisenti, W. Schollkopf, J. P. Toennies, G. C. Hegerfeldt, T. Köhler, and M. Stoll, Phys. Rev. Lett. 85, 2284 (2000).

[7] M. Arndt, O. Nariz, J. Voss-Andreae, C. Keller, G. van der Zouw, and A. Zeilinger, Nature 401, 680 (1999). 
[8] L. Hackermüller, S. Uttenthaler, K. Hornberger, E. Reiger, B. Brezger, A. Zeilinger, and M. Arndt, Phys. Rev. Lett. 91, 090408 (2003).

[9] K. Hornberger and J.E. Sipe, Phys. Rev. A 68, 012105 (2003).

[10] K. Hornberger, J.E. Sipe, and M. Arndt, Phys. Rev. A 70, 053608 (2004).

[11] A. Stibor, K. Hornberger, L. Hackermüller, A. Zeilinger, and M. Arndt Laser Physics 15, 10 (2005)

[12] L. Hackermüller, K. Hornberger, B. Brezger, A. Zeilinger, and M. Arndt, Nature 427, 711 (2004).

[13] K. Hornberger, L. Hackermüller, and M. Arndt, Phys. Rev. A 71, 023601 (2005).

[14] P. Facchi, A. Mariano, and S. Pascazio, Recent Developments in Physics 3, 1 (2002).

[15] R. Alicki, Phys. Rev. A 65, 034104 (2002).

[16] G. Hegerfeldt and T. Köhler, Phys. Rev. Lett. 84, 3215 (2000).

[17] E. H. Wichmann, Am. J. Phys. 33, 20 (1965).

[18] B.-G. Englert, Phys. Rev. Lett. 77, 2154 (1996)

[19] S. Dürr, T. Nonn, and G. Rempe, Phys. Rev. Lett. 81, 5705 (1998). 